\title{
Management Strategies for Diastasis Recti
}

\author{
Maurice Y. Nahabedian, MD, FACS ${ }^{1}$ \\ ${ }^{1}$ National Center for Plastic Surgery, McLean, Virginia \\ Semin Plast Surg 2018;32:147-154.
}

\begin{abstract}
Address for correspondence Maurice Y. Nahabedian, MD, National Center for Plastic Surgery, 7601 Lewinsville Dr., \#400, McLean, VA 22102 (e-mail: DrNahabedian@aol.com).
\end{abstract}

\begin{abstract}
Keywords

- Diastasis recti

- rectus diastasis

- abdominal bulge

- abdominal hernia

- pregnancy

Diastasis recti is a relatively common condition in which there is a midline abdominal bulge that can affect a variety of individuals. The etiology and diagnosis is well understood and optimal management depends on the degree of severity. Patients at high risk for diastasis recti include multiparous women, obese patients, and those with multiple previous operations. Diagnosis includes clinical examination and assessment of symptoms. Physical characteristics include a midline abdominal bulge without a fascial defect. Classification systems are based on the degree of separation between the paired midline rectus and myofascial deformity. Optimal management varies and includes simple plication of the midline defect, extensive plication of the anterior abdominal wall, and sometimes the use of resorbable or nonresorbable mesh.
\end{abstract}

Diastasis recti is a common contour abnormality affecting the anterior abdominal wall. ${ }^{1}$ It is often associated with a negative body image, musculoskeletal pain, and occasionally urogynecological symptoms. ${ }^{2}$ Diastasis recti manifests as a midline abdominal bulge that is the result of an attenuated linea alba. In severe cases, diastasis recti can involve the entire anterior abdominal wall., ${ }^{3,4}$ This condition is most commonly seen in women following pregnancy, obese patients, as well as patients that have had prior abdominal surgery. It is important to differentiate diastasis recti from an abdominal hernia. A true abdominal hernia is characterized by having a fascial defect with protrusion of the abdominal viscera or omentum. A diastasis recti does not have a true fascial defect because the bulge is solely due to the attenuation of the midline linea alba. This review will focus on the etiology, diagnosis, and management of diastasis recti.

\section{Anatomy}

The supportive structures of the anterior abdominal wall include the linea alba, anterior rectus sheath, posterior rectus sheath, external oblique fascia, as well as the paired rectus abdominis and oblique musculature (-Fig. 1). The anterior rectus sheath and the linea alba are composed of collagen fibers arranged in an interwoven lattice. The width of the linea alba ranges from 11 to $21 \mathrm{~mm}$ between the xyphoid process and the umbilicus and decreases from 11 to $2 \mathrm{~mm}$ from the umbilicus to the pubic symphysis. ${ }^{5}$ The thickness of the linea alba ranges from 900 to $1,200 \mu \mathrm{m}$ between the xyphoid and the umbilicus and increases from 1,700 to $2,400 \mu \mathrm{m}$ from the umbilicus to the pubic symphysis. The thickness of the anterior rectus sheath ranges from 370 to $500 \mu \mathrm{m}$ from the xyphoid to the umbilicus and increases to 500 to $700 \mu \mathrm{m}$ from the umbilicus to the pubic symphysis. The posterior rectus sheath is slightly thicker than the anterior rectus sheath above the umbilicus 450 to $600 \mu \mathrm{m}$ but is thinner from the umbilicus to the arcuate line 250 to $100 \mu \mathrm{m}$.

\section{Etiology}

Diastasis recti occurs due to increasing intra-abdominal pressure in which the forces applied to the linea alba cause it to stretch resulting in a widening of the interrectus distance. Most studies have agreed that the minimum interrectus distance to designate a diastasis is $2 \mathrm{~cm} .{ }^{6}$ Diastasis recti is most common following pregnancy; however, obesity and prior abdominal operations can also be the cause. ${ }^{7}$ Studies have demonstrated that the myofascial laxity associated with diastasis recti is both vertical and horizontal and in severe cases can involve the entire anterior abdominal wall including the linea alba and the linea semilunares. ${ }^{3,4,8}$ In a study of 92 women with diastasis recti, the interrecti distance was measured and demonstrated that stretching of the linea alba was limited to $5 \mathrm{~cm}$ in $82 \%$ of patients and can extend up to $6 \mathrm{~cm}$ in $2 \%{ }^{8}$ Abdominal laxity beyond that is usually due to attenuation of the anterior rectus sheath.
Issue Theme Abdominal Wall Reconstruction; Guest Editor: Jesse C. Selber, MD, MPH, FACS
Copyright (C) 2018 by Thieme Medical Publishers, Inc., 333 Seventh Avenue, New York, NY 10001, USA. Tel: +1(212) 584-4662.
DOI https://doi.org/ 10.1055/s-0038-1661380. ISSN 1535-2188. 


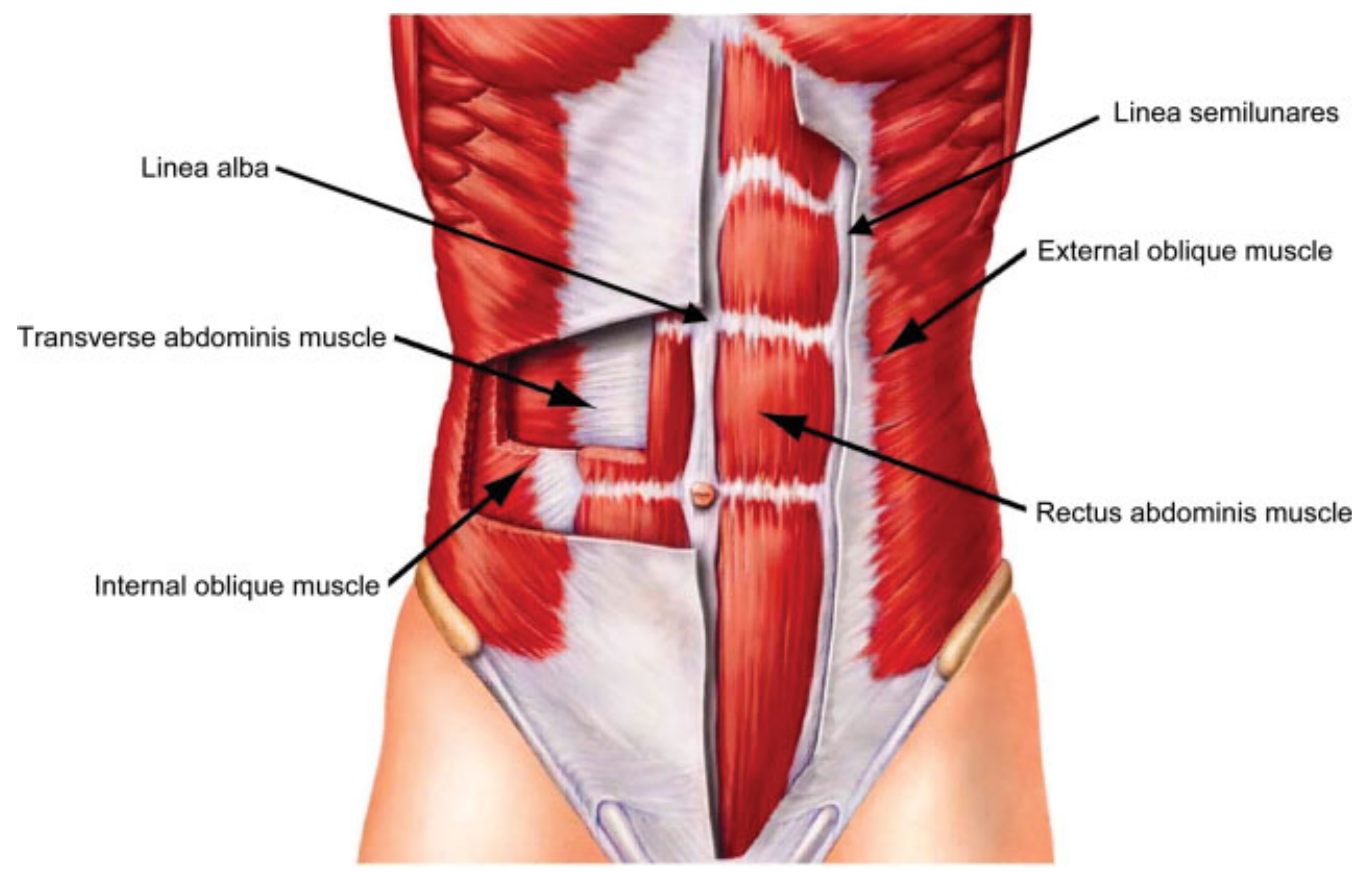

Fig. 1 Illustration of the anterior abdominal wall demonstrating the anterior rectus sheath, linea alba, linea semilunares, and the ventral muscles.

In another study that compared the interrectus distance between nulliparous women and postpartum women using ultrasound, it was demonstrated that postpartum women exhibited a doubling of the interrectus distance from approximately 0.5 to $1.0 \mathrm{~cm}$ to 1.2 to $2.3 \mathrm{~cm}^{7}$ In the postpartum group, there was a gradual decrease in the interrectus distance over time; however, baseline values were never achieved at 6-month assessments. Pregnancy also has a notable effect on the strength of the abdominal musculature with nulliparous women having $5 / 5$ strength of the trunk flexors and rotators compared with $4 / 5$ in women who were 6 months postpartum.

\section{Diagnosis}

The diagnosis of rectus recti is made on the history and physical examination. The presence of a midline abdominal

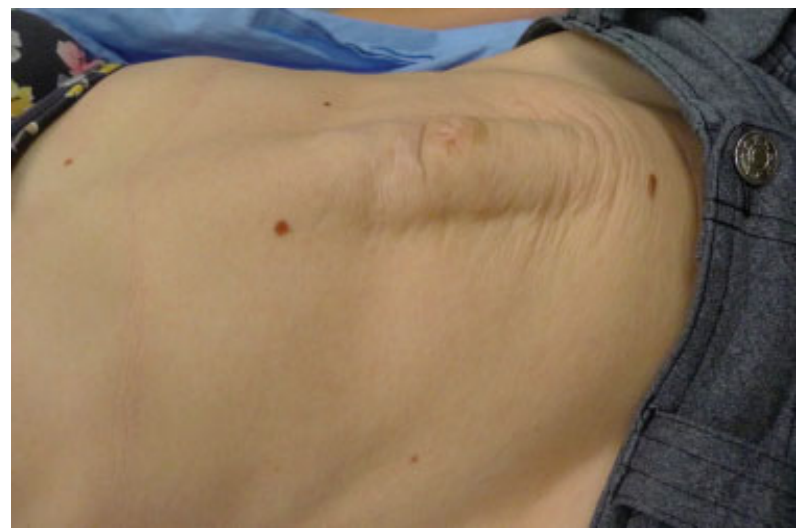

Fig. 2 A woman with rectus diastasis is depicted demonstrating the midline bulge. bulge following pregnancy is usually diagnostic (-Fig. 2). Physical examination can confirm the diagnosis based on a midline bulge above or below the umbilicus that is amplified by having the patient lie flat and perform a straight leg raise. Confirmation of rectus diastasis can be made using computed tomography (CT), magnetic resonance imaging, or ultrasound but these tests are usually not necessary. ${ }^{9-11}$ These imaging modalities are useful to measure the interrectus distance preoperatively; however, they can also be used postoperatively to assess the success of the repair.

\section{Classification}

There are three classifications systems that have been described for rectus diastasis. The Nahas classification is based on the myofascial deformity and the etiology ${ }^{12}$ (-Table 1). The Rath classification is based on the level of the attenuation relative to the umbilicus and the patient age $^{13}$ (-Table 2). The Beer classification is based on the

Table 1 The Nahas classification based on the myofascial deformity

\begin{tabular}{|c|c|c|}
\hline \multicolumn{3}{|c|}{ Nahas classification } \\
\hline Deformity & Etiology & Correction \\
\hline Type A & Pregnancy & Anterior sheath plication \\
\hline Type B & $\begin{array}{l}\text { Myoaponeurotic } \\
\text { laxity }\end{array}$ & External oblique plication \\
\hline Type $\mathrm{C}$ & Congenital & $\begin{array}{l}\text { Rectus abdominis } \\
\text { advancement }\end{array}$ \\
\hline Type D & Obesity & $\begin{array}{l}\text { Anterior sheath plication } \\
\text { and rectus abdominis } \\
\text { advancement }\end{array}$ \\
\hline
\end{tabular}


Table 2 The Rath classification based on the level of the attenuation relative to the umbilicus and the patient age

\begin{tabular}{|c|c|c|}
\hline \multicolumn{3}{|l|}{ Rath classification } \\
\hline Level & Age $<45$ & Age $>45$ \\
\hline Above umbilicus & $10 \mathrm{~mm}$ & $15 \mathrm{~mm}$ \\
\hline At umbilicus & $27 \mathrm{~mm}$ & $27 \mathrm{~mm}$ \\
\hline Below umbilicus & $9 \mathrm{~mm}$ & $14 \mathrm{~mm}$ \\
\hline
\end{tabular}

Table 3 The Beer classification based on the normal width of the linea alba

\begin{tabular}{|l|l|}
\hline \multicolumn{2}{|l|}{ Beer classification } \\
\hline Normal width of the linea alba $(\mathrm{mm})$ \\
\hline Level & Width \\
\hline At Xiphoid & 15 \\
\hline $3 \mathrm{~cm}$ above umbilicus & 22 \\
\hline $2 \mathrm{~cm}$ below umbilicus & 16 \\
\hline
\end{tabular}

normal width of the linea alba as determined from 150 nulliparous women ${ }^{14}$ (-Table 3 ).

\section{Indications for Surgery}

The indications for repair in patients with diastasis recti are based on symptoms and physical findings. ${ }^{15,16}$ Many patients with diastasis recti will have discomfort at the level of the defect that is exacerbated with movement. In addition, the appearance of the abdominal wall is noticeably distorted in patients with diastasis recti especially when there is contraction of the rectus abdominis muscles. An umbilical hernia is often associated with diastasis recti due to the progressive laxity of the midline fascia. Correction of an umbilical hernia without correction of the diastasis is often associated with recurrence due to the poor quality of the surrounding tissue.

\section{Treatment}

There are several options for the management of diastasis recti ranging from exercise to simple plication of the linea alba and anterior rectus sheath to more advanced excisional techniques with or without the use of a mesh. Endoscopic and laparoscopic techniques can also be used in select situations where a small midline hernia is present as well. In many cases, an abdominoplasty is also performed.

\section{Exercise}

The benefit of exercise to prevent or correct diastasis recti is somewhat controversial and has been associated with mixed results. ${ }^{1,17,18}$ Preventative exercise protocols include walking and abdominal core strengthening. Corrective exercise protocols include core strengthening, aerobic activity, and neuromuscular reeducation. Although mild benefit was noted in terms of interrectus distance from some studies, there was insufficient evidence to recommend exercise or physiotherapy programs as a means of preventing or treating rectus diastasis.

Further research with neuromuscular electrical stimulation (NMES) in conjunction with exercise has demonstrated benefit. ${ }^{18}$ In a randomized study of 57 postpartum women with diastasis recti, two cohorts were created and included patients receiving postpartum exercise and $\operatorname{NMES}(n=28)$ and patients receiving postpartum exercise only $(n=29)$. Outcome measures included body mass index (BMI), waist/hip ratio, interrecti distance, and abdominal muscle strength. Both groups demonstrated improvement in all outcomes $(p<0.05)$; however, intergroup comparisons demonstrated significant improvement for all parameters except BMI in favor of women receiving exercise and NMES $(p<0.05)$.

\section{Abdominoplasty}

In most women with mild to severe diastasis recti due to pregnancy, an abdominoplasty is typically performed to further improve the abdominal contour. ${ }^{19-21}$ The salient aspects of an abdominoplasty will be reviewed. The anterior superior iliac crest is palpated and marked bilaterally. A curved low transverse line is drawn connecting the two points with the midpoint just above the pubic hairline. The incision is then created and extends to the level of the anterior rectus sheath. The adipocutaneous tissues are elevated off the anterior rectus sheath preserving the loose areolar layer. The abdominoplasty can be mini and extend to the level of the umbilicus or full and extend to the xyphoid process. The diastasis repair is performed utilizing a variety of techniques that will be described. Following the repair, the patient is gently flexed at the hip and the excess skin is redraped and then excised. Closed suction drains are sometimes utilized and the skin is closed with an absorbable subcuticular suture technique.

\section{Plication}

For mild to moderate diastasis recti, midline plication of the linea alba can be considered. With this technique, the attenuated linea alba is delineated. Following this, a twolayer plication can be achieved using an absorbable or nonabsorbable suture. The triangular suture technique incorporating the lateral edges of the fascia and the midline of the posterior rectus sheath is frequently used. ${ }^{22}$

Studies evaluating absorbable and nonabsorbable sutures have demonstrated no significant difference in the interrecti distance as measured by CT scan at 6 months following correction. ${ }^{23}$ The first layer of sutures was usually an interrupted figure-of- 8 and the second layer of suture was a running continuous to reinforce the repair and to bury to suture knots from the first layer. In patients with significant laxity of the anterior rectus sheath, lateral plication can also be performed on both sides to further improve and tighten the abdominal contour. A two-layer repair technique is usually performed using an absorbable interrupted suture followed by a running continuous suture for further reinforcement. The length of this repair can extend from approximately $2 \mathrm{~cm}$ below the costal margin to approximately $2 \mathrm{~cm}$ above the pubic bone. 
A randomized controlled study compared the time required and the efficiency of plication using three different suture techniques. ${ }^{24}$ In the control group, the anterior rectus sheath was plicated in two layers using 2-0 monofilament nylon suture (control group). Group 1 was plicated with a continuous 2-0 monofilament nylon suture and group 2 was plicated with a continuous barbed suture. The authors demonstrated a significant difference $(p<0.001)$ in mean operative time between the control group (35 minutes) and study groups (group 1, 14 minutes; group 2, 15 minutes). Postoperative ultrasound demonstrated three recurrences in the barbed suture cohort and no differences in the tensile strength of the aponeurosis between the groups.

\section{Plication and Onlay Mesh}

The use of a mesh can be considered in cases of extensive laxity requiring more than simple plication. ${ }^{3,4,19}$ Typically, a resorbable or nonresorbable mesh is selected and placed over the anterior rectus sheath and trimmed to fit the dimensions of the anterior abdominal wall extending from the costal margin superiorly to the pubic region inferiorly and to the anterior axillary line bilaterally. The edge of the mesh is typically anchored in an interrupted manner using an absorbable suture and the central portion of the mesh is secured in a quilting pattern also using an interrupted absorbable suture. A single closed suction drain is usually used.

-Figs. 3-11 illustrate a multiparous woman with severe rectus diastasis and skin laxity (-Figs. 3 and $\mathbf{4}$ ). The plan is to perform an abdominoplasty and repair of the diastasis. The lower abdominal skin is marked and incised extending from one anterior superior iliac crest to the other. Dissection proceeds to the anterior rectus sheath and then extends in

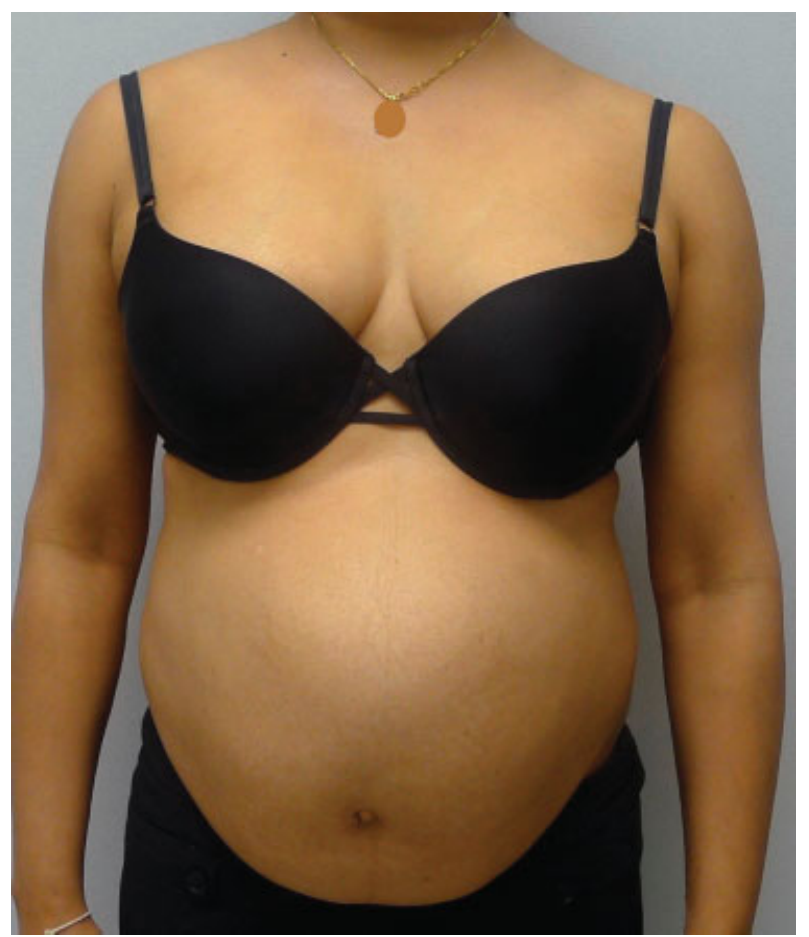

Fig. 3 Preoperative image of a multiparous woman with diastasis recti.

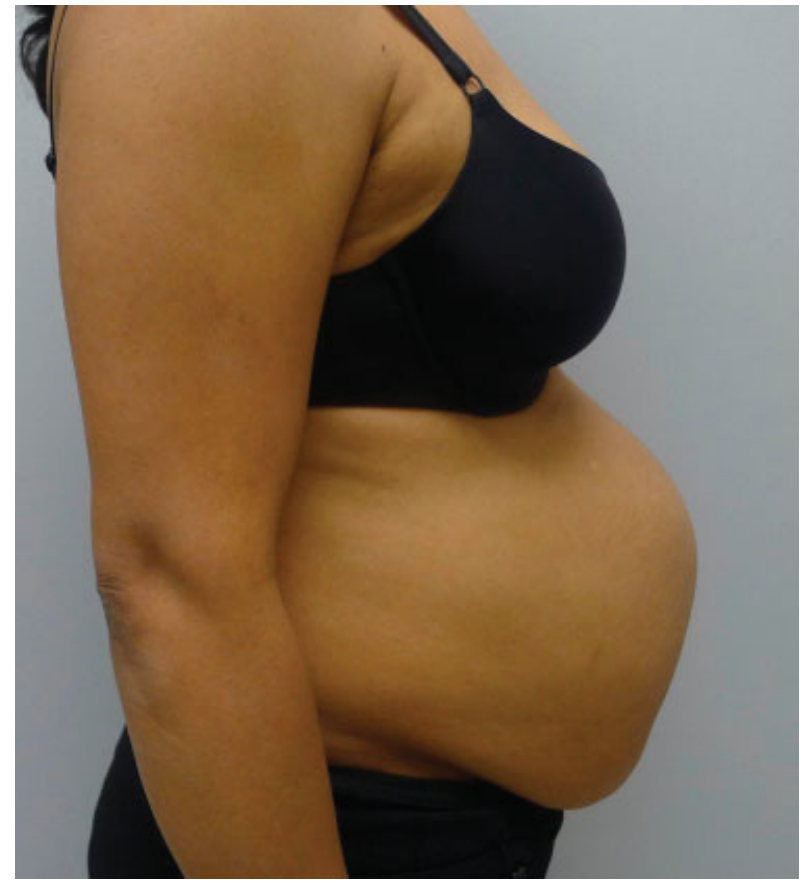

Fig. 4 Lateral view demonstrating significant abdominal laxity and bulge.

a cephalad direction toward the xyphoid process preserving the loose areolar layer and exposing the fascial deformity (-Figs. 5 and $\mathbf{6}$ ). The attenuated midline fascia is delineated and then plicated using the two-layer technique ( - Fig. 7). A permanent or resorbable mesh can be placed as an onlay and secured with absorbable sutures peripherally as well as centrally in a quilting fashion (-Fig. 8). The abdominoplasty is performed by assessing the amount of skin redundancy followed by the adipocutaneous excision (-Fig. 9). Sixmonth follow-up demonstrates a significant improvement in abdominal contour without recurrence (-Figs. 10 and 11).

\section{Retrorectus Repair with Mesh}

In cases of moderate to severe diastasis recti, a retrorectus repair can be considered. ${ }^{25,26}$ With this technique, an

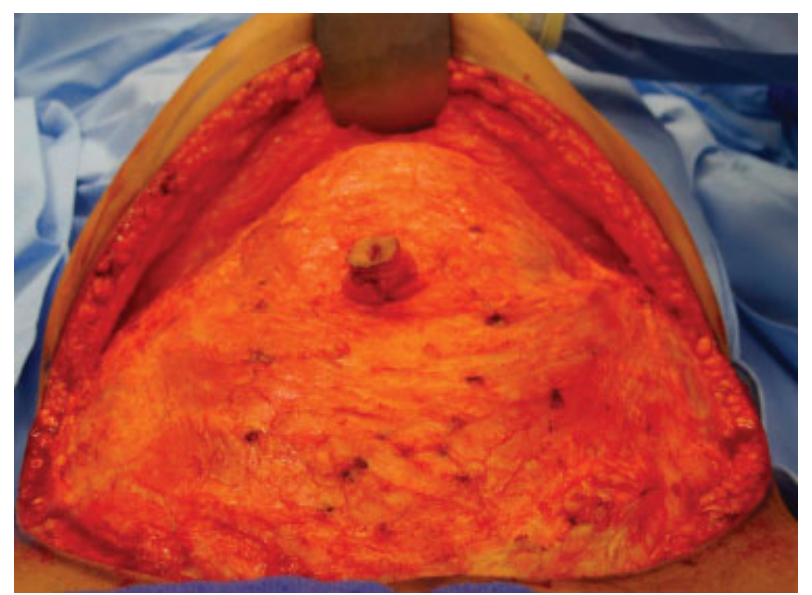

Fig. 5 Intraoperative photograph demonstrating the midline bulge of the linea alba. 


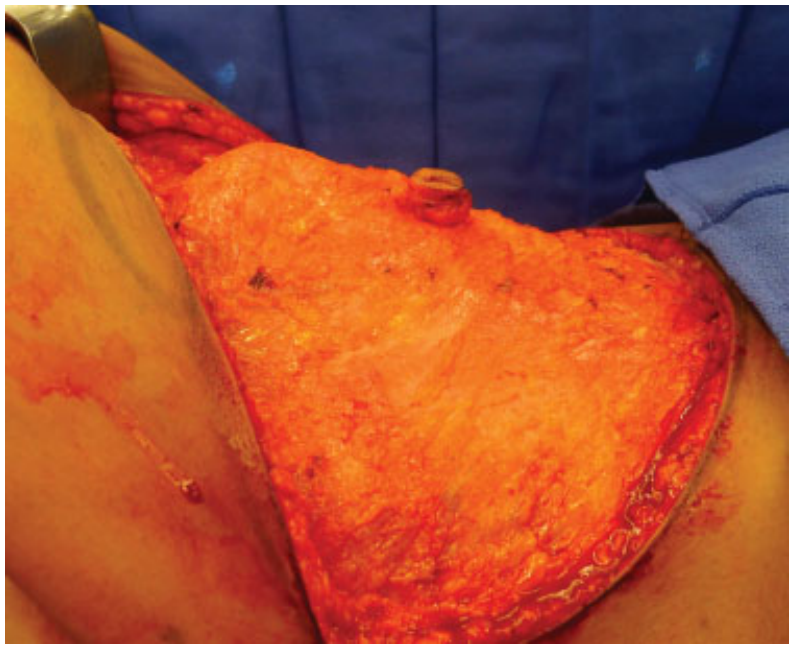

Fig. 6 Intraoperative lateral photograph demonstrating the degree of abdominal protrusion prior to repair.

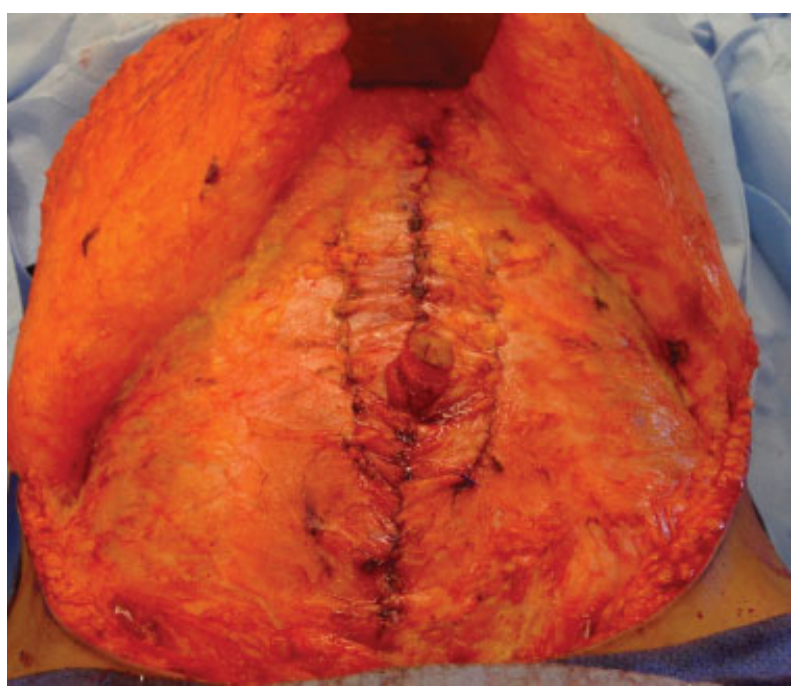

Fig. 7 Intraoperative photograph following three-column plication of the linea alba and anterior rectus sheath using a two-layer technique.

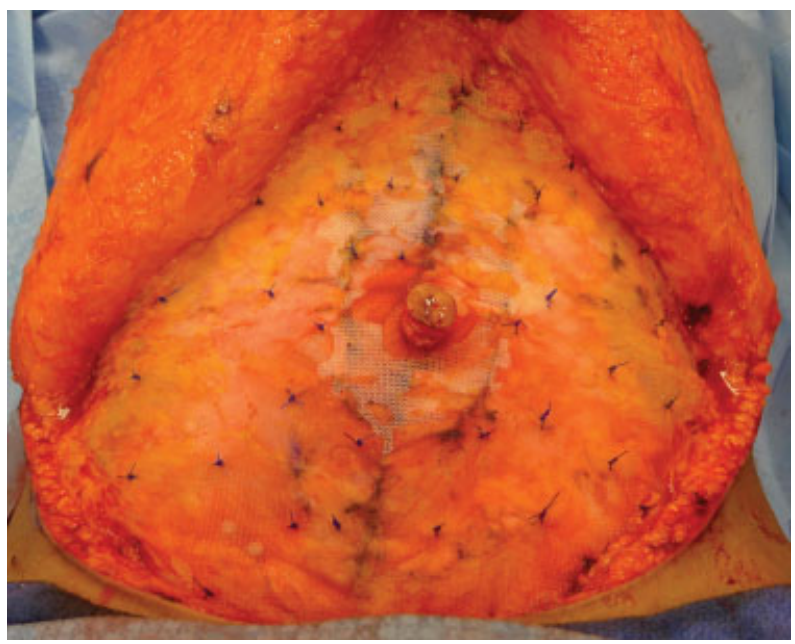

Fig. 8 Intraoperative photograph following placement of the nonresorbable mesh over the plicated anterior rectus sheath.

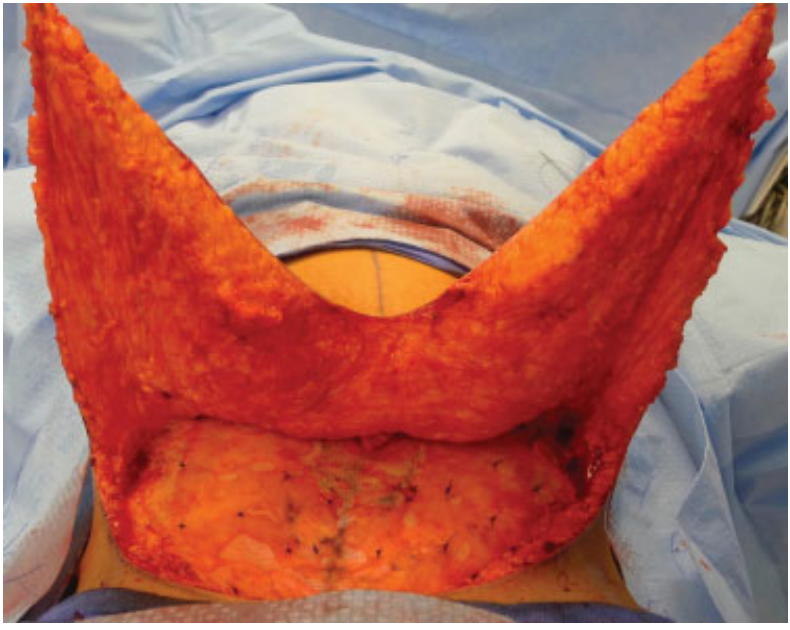

Fig. 9 Intraoperative photograph of the redundant skin and fat constituting the abdominoplasty.

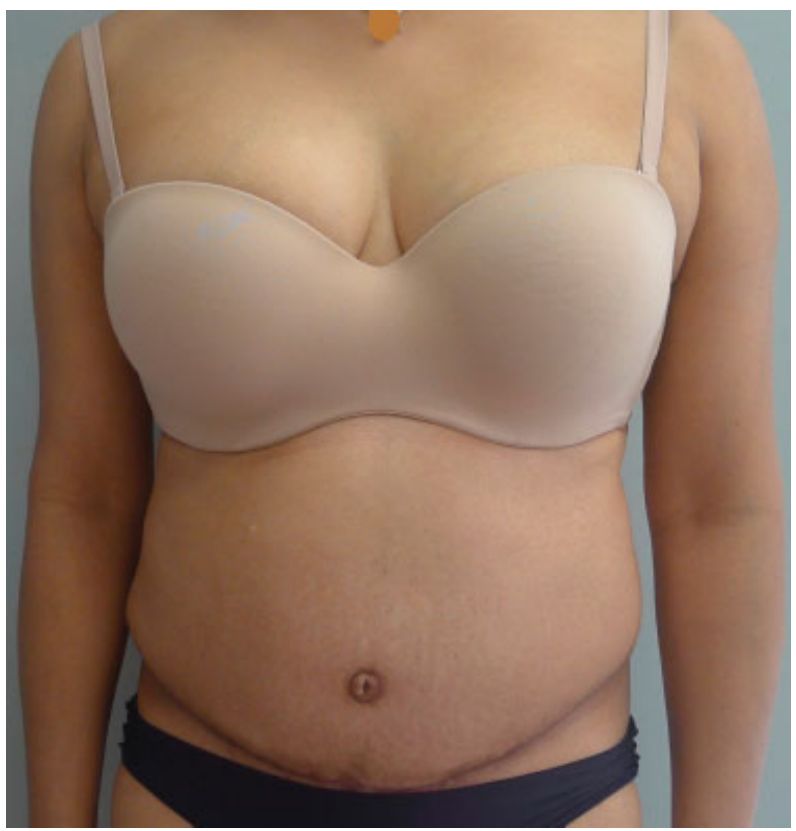

Fig. 10 Six-month postoperative anterior view following successful diastasis repair and abdominoplasty.

anterior paramedian incision is made adjacent to the lateral aspect of the linea alba extending from the xiphoid to the pubic bone. The medial aspect of the rectus abdominis muscle is undermined preserving the vascularity and laterally based innervation. The rectus abdominis muscle is completely released from the posterior rectus sheath. The degree of redundancy of the posterior rectus sheath is approximated and then plicated along its midline using a resorbable suture in an interrupted manner. The repair can then be reinforced using a resorbable or nonresorbable mesh placed on the surface of the posterior rectus sheath to offload the pressure placed on the midline fascial repair. Following the repair, the rectus abdominis muscles are aligned in their natural location. The anterior rectus sheath is repaired using interrupted absorbable sutures. 


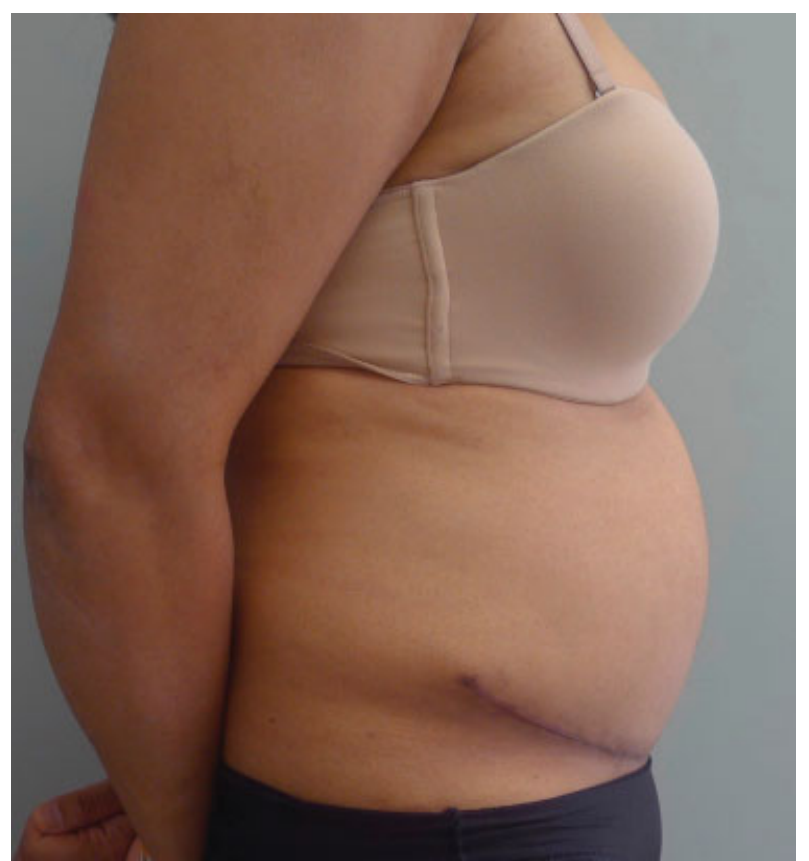

Fig. 11 Six-month postoperative lateral view.

\section{Endoscopic/Laparoscopic}

Endoscopic techniques for repair of diastasis recti in conjunction with umbilical hernia have been described. ${ }^{15}$ The indications for total endoscopic repair include midline/umbilical hernia measuring $>2 \mathrm{~cm}$ and no prior hernia repair or laparotomy. The technique involves placing a trocar into the supra aponeurotic space and creating a dissection plane under direct vision exposing the linea alba and the anterior rectus sheath. The repair includes sheath plication and reinforcement with a synthetic mesh. A nonabsorbable barbed suture is typically used. Adrain is placed and a soft compression garment is applied.

Laparoscopic reinforcement of the anterior abdominal wall can be considered in some patients. In patients who have had plication of the attenuated linea alba and anterior rectus sheath, laparoscopic placement of an intraperitoneal mesh can be considered instead of onlay mesh placement. Huguier et al has applied this technique in 15 women with good to excellent results in 13 of 15 (87\%). ${ }^{27}$

\section{Complications}

Complications following rectus diastasis repair are infrequent and include infection, mesh extrusion, recurrence, nerve injury, seroma, complex scar, skin necrosis, contour abnormality, and visceral injury. In a randomized controlled trial comparing outcomes and complications in women with rectus diastasis managed with layered closure of the anterior rectus sheath or retrorectus placement of synthetic mesh, the incidence of superficial wound infection occurred was 14 in 57 (24.5\%) of which 5 of 57 (8.8\%) were in the suture repair cohort and 9 of $57(15.8 \%)$ in the retrorectus mesh cohort. ${ }^{28}$ Postoperative pain was less in the retrorectus cohort (6.9) compared with the sheath plication cohort (4.8).

\section{Outcomes}

\section{Sheath Plication}

The outcomes following sheath plication for diastasis recti have been mixed and primarily related to the type of suture used for the plication. al-Qattan in a review of 20 women following vertical sheath plication alone using an absorbable suture demonstrated $100 \%$ recurrence after 1 year. ${ }^{29}$ Reasons included a repair that was localized to the defect only, a repair that addressed only the horizontal component of the diastasis, and suture-related fraying of the anterior rectus sheath. Nahas et al using a nonabsorbable suture had positive outcomes utilizing a two-layer plication repair. ${ }^{10}$ Efficacy of the repair was evaluated by postoperative CT scans in 12 women at 3 weeks, 6 months, and again at a mean of 81 months. The interrectus distance was measured $3 \mathrm{~cm}$ above and below the umbilicus. No recurrence of diastasis rectus was demonstrated in any patient at all levels studied. Mestak et al performed a case-controlled study comparing 51 women that had diastasis recti repair via plication with an interlocking continuous absorbable suture (0-PDS) to 10 nulliparous women without a diastasis. ${ }^{9}$ Postoperative assessment was performed at 12 to 41 months using ultrasound and physical examination. The mean interrecti distance was essentially equal between the two cohorts at all levels studied. The use of absorbable sutures was recommended to avoid long-term palpability issues.

The type and orientation of suture material used for diastasis repairs has also been comparatively studied. Nahas et al has compared diastasis repair techniques using absorbable (0-polydioxanone) sutures to nonabsorbable (2-0 nylon) sutures. ${ }^{23} \mathrm{CT}$ scans obtained at 3 weeks and 6 months demonstrated no significant difference between the two suture techniques. ${ }^{16}$ Ishida et al in a cadaveric study compared the strength of a horizontal versus vertical suture repair using a dynamometer. ${ }^{30}$ The strength required for disrupting the vertical repair was increased relative to the horizontal, thus vertical orientation was recommended.

\section{Retrorectus Repair}

The role of mesh placement in the retrorectus location is to redistribute the forces placed on the posterior sheath repair and to reduce the risk of recurrence. Batchvarova et al have utilized this technique in 52 women with up to 11 years of follow-up ${ }^{25}$ using a vicryl mesh. According to Batchvarova et al, a resorbable mesh is preferred because it effectively relieves fascial tension, is resorbed by 6 weeks, is placed in an extraperitoneal position, and does not increase the incidence of complications.

In the Emanuelsson et al study, 36-Item Short Form Health Survey outcomes were compared following repair via anterior sheath plication versus retrorectus mesh placement. ${ }^{28}$ The results at 3-month follow-up demonstrated improvement in both cohorts following the repair with no technique demonstrating superiority over the other. Subjective improvement in muscle strength was improved more in the retrorectus cohort compared with the suture cohort ( 6.9 vs. 4.5 , Likert scale, $0-10$, $p=0.01$ ). Longer follow-up at 1 year demonstrated no difference between the two cohorts with significant improvements in abdominal wall stability, strength, and pain. ${ }^{31}$ 


\section{Endoscopic/Laparoscopic}

The most frequent adverse event with the endoscopic technique is seroma (23\%). ${ }^{15}$ In the 21 patients from the Bellido Luque et al study, there were no hernia or diastasis recurrences at 20-month follow-up. ${ }^{15}$ The mean interrectus distance was significantly improved at 1 month following the procedure with preoperative measurements ranging from 24 to $39 \mathrm{~mm}$ and postoperative measurements ranging from 2.1 to $2.8 \mathrm{~mm}$. One- and 2-year follow-up did not change from the 1 month measurements (2.5-3.7 mm). Patient satisfaction was assessed on a visual analog scale and graded with a mean score of 8.7.

\section{Conclusion}

The etiology, diagnosis, and management of diastasis recti are well understood and have demonstrated success. Multiparous women are at highest risk for developing diastasis recti. Diagnosis is easily made by clinical examination and symptomology. Management options vary and will depend on the degree of separation between the rectus abdominis muscles as well as the flaccidity of the anterior abdominal wall. Simple plication has been effective for mild to moderate diastasis. The use of resorbable or nonresorbable mesh placed as an onlay or in the retrorectus space has been effective for moderate to severe diastasis.

\section{References}

1 Mommers EHH, Ponten JEH, Al Omar AK, de Vries Reilingh TS, Bouvy ND, Nienhuijs SW. The general surgeon's perspective of rectus diastasis. A systematic review of treatment options. Surg Endosc 2017;31(12):4934-4949

2 Keshwani N, Mathur S, McLean L. Relationship between inter-rectus distance and symptom severity in women with diastasis recti in the early postpartum period. Phys Ther 2018;98(03):182-190

3 Nahabedian MY. Diastasis recti. In: Cobb W, Hope W, eds. Textbook of Hernia. New York, NY: Springer Publishing; 2017

4 Nahabedian MY. Diagnosis and management of diastasis recti. In: Novitsky Y, Henniford T, eds. Current Principles in Surgery of the Abdominal Wall. New York, NY: Springer Publishing; 2016

5 Axer H, Keyserlingk DG, Prescher A. Collagen fibers in linea alba and rectus sheaths. I. General scheme and morphological aspects. J Surg Res 2001;96(01):127-134

6 Nahabedian MY, Brooks DC. Rectus abdominis diastasis. Available at: https://www.uptodate.com/contents/rectus-abdominis-diastasis Updated March 22, 2017

7 Liaw LJ, Hsu MJ, Liao CF, Liu MF, Hsu AT. The relationships between inter-recti distance measured by ultrasound imaging and abdominal muscle function in postpartum women: a 6 -month follow-up study. J Orthop Sports Phys Ther 2011;41(06):435-443

8 Brauman D. Diastasis recti: clinical anatomy. Plast Reconstr Surg 2008;122(05):1564-1569

9 Mestak O, Kullac R, Mestak J, Nosek A, Krajcova A, Sukop A. Evaluation of the long-term stability of sheath plication using absorbable sutures in 51 patients with diastasis of the recti muscles: an ultrasonographic study. Plast Reconstr Surg 2012;130(05): $714 \mathrm{e}-719 \mathrm{e}$

10 Nahas FX, Ferreira LM, Augusto SM, Ghelfond C. Long-term followup of correction of rectus diastasis. Plast Reconstr Surg 2005;115 (06):1736-1741, discussion 1742-1743

11 Elkhatib H, Buddhavarapu SR, Henna H, Kassem W. Abdominal musculoaponeuretic system: magnetic resonance imaging eva- luation before and after vertical plication of rectus muscle diastasis in conjunction with lipoabdominoplasty. Plast Reconstr Surg 2011;128(06):733e-740e

12 Nahas FX. An aesthetic classification of the abdomen based on the myoaponeurotic layer. Plast Reconstr Surg 2001;108(06):1787-1795, discussion 1796-1797

13 Rath AM, Attali P, Dumas JL, Goldlust D, Zhang J, Chevrel JP. The abdominal linea alba: an anatomo-radiologic and biomechanical study. Surg Radiol Anat 1996;18(04):281-288

14 Beer GM, Schuster A, Seifert B, Manestar M, Mihic-Probst D, Weber $\mathrm{SA}$. The normal width of the linea alba in nulliparous women. Clin Anat 2009;22(06):706-711

15 Bellido Luque J, Bellido Luque A, Valdivia J, et al. Totally endoscopic surgery on diastasis recti associated with midline hernias. The advantages of a minimally invasive approach. Prospective cohort study. Hernia 2015;19(03):493-501

16 Nahabedian MY, Nahabedian AG. Closing the gap for patients with rectus abdominis diastasis. Nursing 2018;48(01):49-52

17 Benjamin DR, van de Water ATM, Peiris CL. Effects of exercise on diastasis of the rectus abdominis muscle in the antenatal and postnatal periods: a systematic review. Physiotherapy 2014;100(01):1-8

18 Kamel DM, Yousif AM. Neuromuscular electrical stimulation and strength recovery of postnatal diastasis recti abdominis muscles. Ann Rehabil Med 2017;41(03):465-474

19 Akram J, Matzen SH. Rectus abdominis diastasis. J Plast Surg Hand Surg 2014;48(03):163-169

20 Cardenas Restrepo JC, Munoz Ahmed JA. New technique of plication for miniabdominoplasty. Plast Reconstr Surg 2002;109(03): 1170-1177, discussion 1178-1190

21 Tadiparthi S, Shokrollahi K, Doyle GS, Fahmy FS. Rectus sheath plication in abdominoplasty: assessment of its longevity and a review of the literature. J Plast Reconstr Aesthet Surg 2012; 65(03):328-332

22 Ferreira LM, Castilho HT, Hochberg J, et al. Triangular mattress suture in abdominal diastasis to prevent epigastric bulging. Ann Plast Surg 2001;46(02):130-134

23 Nahas FX, Augusto SM, Ghelfond C. Nylon versus polydioxanone in the correction of rectus diastasis. Plast Reconstr Surg 2001;107 (03):700-706

24 Gama LJM, Barbosa MVJ, Czapkowski A, Ajzen S, Ferreira LM, Nahas FX. Single-layer plication for repair of diastasis recti: the most rapid and efficient technique. Aesthet Surg J 2017;37(06):698-705

25 Batchvarova Z, Leymarie N, Lepage C, Leyder P. Use of a submuscular resorbable mesh for correction of severe postpregnancy musculoaponeurotic laxity: an 11-year retrospective study. Plast Reconstr Surg 2008;121(04):1240-1248

26 Cheesborough JE, Dumanian GA. Simultaneous prosthetic mesh abdominal wall reconstruction with abdominoplasty for ventral hernia and severe rectus diastasis repairs. Plast Reconstr Surg 2015;135(01):268-276

27 Huguier V, Faure JL, Doucet C, Giot JP, Dagregorio G. Laparoscopy coupled with classical abdominoplasty in 10 cases of large rectus diastasis [in French]. Ann Chir Plast Esthet 2012;57(04):350-355

28 Emanuelsson P, Gunnarsson U, Strigård K, Stark B. Early complications, pain, and quality of life after reconstructive surgery for abdominal rectus muscle diastasis: a 3-month follow-up. J Plast Reconstr Aesthet Surg 2014;67(08):1082-1088

29 al-Qattan MM. Abdominoplasty in multiparous women with severe musculoaponeurotic laxity. Br J Plast Surg 1997;50(06):450-455

30 Ishida LH, Gemperli R, Longo MVL, et al. Analysis of the strength of the abdominal fascia in different sutures used in abdominoplasties. Aesthetic Plast Surg 2011;35(04):435-438

31 Emanuelsson P, Gunnarsson U, Dahlstrand U, Strigård K, Stark B. Operative correction of abdominal rectus diastasis (ARD) reduces pain and improves abdominal wall muscle strength: a randomized, prospective trial comparing retromuscular mesh repair to doublerow, self-retaining sutures. Surgery 2016;160(05):1367-1375 